\title{
Altering form for function
}

\section{Elucidating the mechanisms by which biomolecules are chemically modified and how these alterations regulate biological pathways represents a leading frontier in chemical biology.}

The biomolecular inventory of cells includes genetically encoded macromolecules, such as DNA, RNA and proteins, and organic compounds such as lipids, carbohydrates and structurally diverse small-molecule metabolites, which are biosynthesized in sequential enzymatic transformations. Independent of their origins, many biomolecules undergo post-synthetic molecular tailoring reactions in which chemical groups-as small as methyl or phosphate groups and as large as proteins or complex polysaccharides-are attached, typically enzymatically, at precise sites of their structures. In addition to the numerous post-translational modifications (PTMs) that decorate cellular proteins, chemical modifications have been observed in most classes of biomolecules, including DNA and RNA, lipids, carbohydrates and natural products. The presence of these modified biomolecules in cells, along with the proteins that selectively install, revert or bind these chemical marks, have established dynamic biomolecular tailoring as a distinct mechanism of biological regulation.

In this special issue, we present a collection of articles that explores diverse aspects of chemical tailoring in cells, highlighting how chemical biology contributes to our mechanistic understanding of these modified biomolecules and provides tools to illuminate their biological functions.

Protein PTMs, in which specific amino acid side chains are modified with a diverse array of chemical groups, are the most familiar type of biomolecular tailoring reaction. As discussed by Kelleher and an esteemed group of proteomics experts (Perspective, p. 206), the diversity in the structures and modification sites of PTMs, along with many other factors, contributes to a dramatic expansion of the potential size of the proteome. Following a systematic analysis of proteome complexity, the authors estimate that the actual number of 'proteoforms' within cells is likely far below theoretical limits, which enhances prospects for full cellular proteome mapping by mass spectrometry- and chemical biology-based techniques. Given the primary role of proteins in executing the genomic programs of the cell, we believe that it is time to commit to a 'human proteoform project' that will enable a comprehensive analysis of cellular proteomes and their roles in biology and disease.
Barber and Rinehart (Commentary, p. 188) provide compelling arguments that chemical biologists are ideally placed to lead efforts to identify PTMs and to understand their biological roles. In particular, they highlight chemical and biological methods that enable the production of tailor-made modified proteins as tools for chemical biologists to validate the cellular functions and dynamics of PTMs, while at the same time, offering platforms for synthetic biologists for next-generation cellular engineering.

Although most protein-tailoring reactions are enzyme catalyzed, Harmel and Fiedler (Review, p. 244) explore PTMs that result from uncatalyzed reactions between nucleophilic or redox-sensitive amino acids and an activated metabolite. The extent to which these 'spontaneous' reactions serve as markers of cellular stress or as contributors to the cell's steady state PTM levels highlights the importance of defining the cellular mechanisms that control the abundance of individual PTMs.

Along these lines, chemical biologists have been active in identifying and characterizing the client protein and amino acid site specificities of enzymes that biosynthesize or turn over PTMs. For example, two Research Highlights (p. 199) report on mechanistic insights derived from structural studies of an oligosaccharyltransferase for $\mathrm{N}$-glycan biosynthesis and a palmitoyl transferase involved in protein S-lipidation. Similarly, Cohen and Chang (Review, p. 236) examine recent structural and functional insights into ADP-ribosyltransferase (ART) enzymes, which mediate the transfer of ADP-ribose (ADPr) from $\mathrm{NAD}^{+}$to diverse protein substrates in eukaryotes and bacteria. Beyond structural insights, chemical biologists are keenly aware of the need to understand PTM function and metabolism within cells; for example, how PTMs (e.g., ubiquitylation, Research Highlights, p. 199) alter cellular pathways and how native protein states are restored (Commentary, p. 188; Review, p. 244).

In contrast to proteins, nucleic acids are viewed as relatively static structures, but recent studies of DNA and RNA modifications have refined our knowledge of their potential regulatory roles. For example, selective methylation of mammalian DNA and proposed pathways involving oxidative demethylation (Nat. Chem. Biol. 14, 72-78, 2018) have revealed how subtle modifications may yield dramatic effects on gene expression. RNA nucleotides, especially those within rRNA and tRNA, contain numerous chemical modifications. Polikanov and colleagues (Review, p. 226) catalog rRNA methylation and highlight structural studies that have permitted the direct visualization of methylated rRNA residues within bacterial and eukaryotic ribosomes as a means to understand their biosynthesis and functions.

Expanding interest in "RNA epigenetics," in which reversible modifications of cellular RNAs are thought to regulate gene expression at the RNA level (Nat. Chem. Biol. 6, 863$865,2010)$, has led to the development of methods for epitranscriptomic profiling of RNA modifications such as $N^{1}$-methyladenosine $\left(\mathrm{m}^{1} \mathrm{~A}\right)$ and 5 -methylcytidine $\left(\mathrm{m}^{5} \mathrm{C}\right)$, as discussed by Grozhik and Jaffrey (Perspective, p. 215). In light of recent published data, these authors also call for community standards for validation of epitranscriptomic data sets as a means to ensure their reproducibility and robustness.

Informational macromolecules are not the only substrates for biomolecular tailoring. As discussed by Jackson and colleagues (Commentary, p. 193), the cell envelope of mycobacteria contains unique lipopolysaccharides and glycolipids that themselves undergo further modification, such as succinylation and glycosylation with diverse mono- and oligosaccharide donors. However, the functional roles of these modifications remain unclear, prompting the authors to outline research directions that chemical biologists will embrace.

Biosynthetic tailoring reactions are also important for diversifying the structures of small molecules. On some peptidic natural products such as the omphalotins (Nat. Chem. Biol. 13, 833-835, 2017) and divamides (Nat. Chem. Biol. 14, 179-185, 2018), even modifications as small as methyl groups prove essential for endowing the compound with biological activity.

Even as the current issue only excerpts the narrative of an expanding field, we hope that it prompts chemical biologists to apply their mechanistic mindsets and creative toolsets to advance our understanding of the diverse chemistry of biomolecular tailoring and its effects on biological systems. 